\title{
SCHOPENHAUER DANS LE ROMAN. A PROPOS DE SIXTINE DE REMY DE GOURMONT
}

\begin{abstract}
A b stra c t. Malinowski Wieslaw Mateusz, Schopenhauer dans le roman. A propos de «Sixtine» de Remy de Gourmont [Schopenhauer in the novel. On De Sixtine by Gourmont]. Studia Romanica Posnaniensia, Adam Mickiewicz University Press, Poznań, vol. XXIX: 2003, pp. 55-67, ISBN 83-2321232-5, ISSN 0137-2475.

The subject of the paper is the mode of presence of Schopenhauer's philosophical thought in Remy de Gourmont's 1890 novel entitled Sixtine, roman de la vie cérébrale. The pessimistic premisses of the main character's extreme subjectivism are accompanied by an attempt to escape from Schopenhauerian omnipotent will to live (vouloir-vivre) through art, in this case through literary activity. Here originates the characteristic and innovative on the aesthetic ground motif of the novel in the novel in Gourmont's work.
\end{abstract}

En étudiant, il y a plus de vingt ans, la présence de Schopenhauer en France, René-Pierre Colin en a fait essentiellement un «mythe naturaliste»: Zola, Maupassant, Céard et Huysmans lui apparaissaient comme les maîtres du pessimisme, substantiellement nourris par la lecture du philosophe allemand ${ }^{1}$. On ne peut certes que souscrire aux constatations du critique, même si Zola refusera toujours de reconnaître dans son oeuvre la marque de ce qu'il appelle «la grande poésie noire» de Schopenhauer. On observera néanmoins que le Schopenhauer fréquenté par les naturalistes est assez rétréci: à l'exception de Maupassant, qui en a eu une connaissance approfondie, beaucoup se contentaient de lire la présentation qu'en a donnée en 1874 Théodule Ribot dans sa Philosophie de Schopenhauer, ou les traductions fragmentaires qu'apportait en 1881 l'anthologie de Jean Bourdeau, Pensées et fragments, sévèrement jugée aujourd'hui par les spécialistes ${ }^{2}$. C'est cette

${ }^{1}$ R.-P. Col i n, Schopenhauer en France: un mythe naturaliste, Presses Universitaires de Lyon, 1979.

${ }^{2}$ Voir A. He n ry (dir.), Schopenhauer et la crêation littéraire en Europe, Klincksieck, Paris 1989, p. 17 et 32. Sur les traductions françaises de Schopenhauer, voir aussi F.-X. C h e n e t, Traduire Schopenhauer, «Magazine littéraire», janvier 1995, p. 65-66. 
dernière pourtant qui sera le bréviaire du pessimisme pour de nombreux écrivains français, avant que ne soit traduit par Auguste Burdeau, en 1888-1890, Le Monde comme volonté et comme représentation. Aussi toute l'originalité du système schopenhauerien, qui consiste à renverser les valeurs attachées à la connaissance pour faire de limmense force inconsciente du vouloir-vivre le principe du monde, sera-t-elle rarement assimilée par les naturalistes dans sa version intégrale. Elle trouvera en revanche une expression beaucoup plus complète et réfléchie chez un écrivain comme Remy de Gourmont, l'historien et le porte-parole du mouvement symboliste, mais aussi poète, dramaturge et romancier, dans une oeuvre de 1890 qui reste étroitement liée aux années héroïques du symbolisme et qui a pour titre Sixtine, roman de la vie cérébrale.

Nous avons bien affaire ici à ce qu'on pourrait appeler le «roman de l'intellectuel». Hubert d'Entragues, le protagoniste de Sixtine, est un jeune écrivain parisien doté d'une vaste culture et qui accorde une grande valeur à l'intellect. Il fréquente peu le monde, préférant la vie de l'esprit à toute autre forme d'activité:

Paris, ce n'était pour lui, ni la rue, ni le boulevard, ni le théâtre; Paris, pour Entragues, était confiné dans les bornes assez étroites du «cabinet d'étude» peuplé de bons fantômes de son imagination. Là, s'agitaient obscurément des êtres tristes et vagues, pensifs et informes, qui imploraient l'existence. Entragues vivait avec eux dans une familiarité presque inquiếtante ${ }^{3}$.

Dès le début, il nous dit sa volonté d'abolir le monde extérieur:

Je mêprisais tout ce qui m'était extérieur, tout ce qui n'avait pas été rebroyé et repétri par la machine sans cesse en mouvement dans ma tête (p. 62).

Cette espèce de désintéressement total de la réalité historique contemporaine pousse le héros de Sixtine à se tourner vers une forme radicale de l'individualisme, celle qui, en vertu d'une vision substantiellement solipsiste du monde, en fait «ma représentation». Et c'est là ce qui donne au livre de Gourmont sa dimension et sa tonalité particulières. Avec sa conception élitiste de l'homme de pensée, avec sa mince intrigue qui n'est qu'un prétexte à entraîner le lecteur parmi les méditations et les systèmes, le roman prend une orientation résolument philosophique.

Dans ses écrits, Remy de Gourmont se réclame explicitement, et à maintes reprises, de la philosophie idéaliste. "A cette heure, la théorie idéaliste n'est plus guère contestée que par quelques canards enclins à se plaire dans les vieux marécages», note-t-il en 1893 dans une phrase propre à faire rentrer sous terre les survivants du naturalisme ${ }^{4}$. Cependant, par «idéalisme» il entend avant tout, précisément, la doctrine pessimiste de Schopenhauer. On découvre dans Sixtine

${ }^{3}$ R. de G o u r m o n t, Sixtine. Roman de la vie cérébrale, Union Générale d'Éditions, Paris 1982, coll. 10/18, série «Fins de siècles», p. 55. C'est à cette édition que renvoient dans le présent article toutes les références des citations.

${ }^{4}$ R. de G o u r m o n t, L'Idéalisme, Mercure de France, Paris 1893, p. 7. 
(chap. XIV et XVI) plusieurs références explicites à Théodule Ribot, ce qui prouve déjà que Gourmont connaissait son ouvrage de vulgarisation, La Philosophie de Schopenhauer. Il écrira plus tard:

A vrai dire, notre éducation philosophique à quelques - uns avait (...) été faite par le Schopenhauer de M. Bourdeau et celui de M. Ribot. Nous avions déjà découvert, et avec quelle ivresse, à la fois que le monde était mauvais et qu'il n'existait que relativement à nous-mêmes ${ }^{5}$.

Dans Le Livre des Masques, Gourmont s'affirmera ouvertement comme schopenhauerien, en parlant d'une vérité nouvelle entrée récemment dans la littérature et dans l'art:

Cette vérité, évangélique et merveilleuse, libératrice et rénovatrice, c'est le principe de l'idéalité du monde. Par rapport à l'homme, sujet pensant, le monde, tout ce qui est extérieur au moi, n'existe que selon l'idée qu'il s'en fait. Nous ne connaissons que des phénomènes, nous ne raisonnons que sur des apparences; toute vérité en soi nous échappe; l'essence est inattaquable. C'est ce que Schopenhauer a vulgarisé sous cette formule si simple et si claire: Le monde est ma représentation. Je ne vois pas ce qui est; ce qui est, c'est ce que je vois ${ }^{6}$.

Dès 1890 , cependant, toute l'architecture romanesque de Sixtine s'organise déjà autour de ce principe. La théorie de l'idéalisme transcendental à la Schopenhauer s'y trouve exposée par fragments dont il est aisé, pour le lecteur un peu attentif, de faire la synthèse. En évoquant la place et les modalités du fonctionnement de la pensée de Schopenhauer dans Sixtine, André Karátson est allé jusqu'à exprimer la conviction selon laquelle l'intérêt de Sixtine réside précisément dans la réception de Schopenhauer: «Lecture créatrice de la doctrine, le roman transforme le fameux postulat: Le monde est ma représentation en le monde est la représentation de moi-même», affirmait le critique. Cette modification, ajoutait-il, indépendamment d'une certaine dose d'ironie qu'elle laisse deviner à l'égard du penseur allemand, engage tout un programme esthétique qui équivaut non seulement à la rupture symboliste avec le naturalisme, mais encore, sous certains aspects du moins, à l'ouverture vers des expériences esthétiques modernes ${ }^{7}$.

On ne saurait mieux dire. Il importe toutefois, pour miex comprendre la dimension philosophique du roman et les conséquences artistiques qui en découlent, d'observer la chose de plus près.

La formule utilisée par Gourmont dans le sous-titre de son roman semble véhiculer déjà une première référence à Schopenhauer. L'auteur du Monde comme

${ }^{5}$ R. de G o u r m o n t, Promenades littéraires, $4^{e}$ série, Mercure de France, Paris 1927, p. 71-72.

${ }^{6} \mathrm{R}$. de G o u r m o n t, Le Livre des Masques, Mercure de France, Paris 1896, p. 11-12.

${ }^{7} \mathrm{~A}$. Karáts on, Les arcanes de l'idéalisme (Réception esthêtique de Schopenhauer dans «Sixtine» de Remy de Gourmont), đans: A. H e n r y (dir.), Schopenhauer et la création littéraire en Europe, Klincksieck, Paris 1989, p. 116. 
volonté et comme représentation expliquait bien que «ce monde réel, intuitif est manifestement un phénomène du cerveau» et que par conséquent «l'hypothèse qu'il puisse y avoir un monde, en tant que tel, en dehors de tout cerveau, est contradictoire». Il en va exactement ainsi pour le protagoniste de Gourmont. Dès sa première rencontre avec Sixtine, jeune veuve dont il tombera amoureux, Hubert d'Entragues se livre devant elle à une méditation qui ressemble fort à une profession de foi:

- Si je ne suis pas mon propre juge, qui me jugera, et si je me déplais à moi-même que m'importe de plaire à autrui? Quel autrui? Y a-t-il un monde de vie extérieure à moi-même? C'est possible, mais je ne le connais pas. Le monde, c'est moi, il me doit l'existence, je l'ai créé avec mes sens, il est mon esclave et nul sur lui n'a de pouvoir. Si nous étions bien assurés de ceci, qu'il n'est rien en dehors de nous, comme la guérison de nos vanités serait prompte, comme promptement nos plaisirs en seraient purgés. La vanité est le lien fictif qui nous annexe à une extériorité imaginaire: un petit effort le brise et nous sommes libres! Libres, mais seuls, seuls, dans l'effroyable solitude où nous naissons, où nous vivons, où nous mourrons (p. 51).

À quoi Sixtine réagit par cette exclamation:

- Quelle triste philosophie, mais quel orgueil! (...). Qui vous a induit là?

Plutôt que d'indiquer sa source, Hubert se contente de déclarer:

- Mais c'est naturel, comment concevoir une vie différente de ce qu'elle apparât clairement à tout oeil qui sait regarder? (p. 51).

Schopenhauer sera pourtant nommé et même cité en exergue au chapitre VIII; Gourmont y rapporte le passage du Monde comme volonté et comme représentation relatif au Cogito cartésien:

En posant son Cogito ergo sum comme seul certain et en considérant l'existence du monde comme problématique, Descartes a trouvé le point de départ essentiel de toute philosophie (Le monde, I, livre I,8; Sixtine, p. 88).

On retrouvera cet éloge de Descartes quelques pages plus loin, dans les pensées du protagoniste:

Entragues aimait le voisinage des livres qui lui démontraient la probabilité de sa philosophie. Il ne se lassait pas d'admirer la courageuse persêvếrance des hommes à redire toujours la même chose. Tout ce qui avait été rédigé depuis l'Écriture se pouvait résumer en trois mots; flambés en un fantastique creuset, la totalité des livres donnerait pour résidu chimique: COGITO, ERGO SUM: Descartes était le seul homme qui eût jamais écrit une parole nécessaire et treize lettres y suffisaient. Il aurait voulu les voir gravées au front des monuments (p. 92).

${ }^{8} \mathrm{~A}$. S $\mathrm{ch}$ o p e $\mathrm{nh}$ a u $\mathrm{r}$, Le monde comme volonté et comme représentation (Supplèment au livre premier, première partie, chap. I), traduit en français par A. Burdeau, PUF, Paris 1966, p. 674 et passim. 
Il est intéressant de rapprocher ce passage de Sixtine du commentaire que fera Gourmont dans un autre texte, à propos de Schopenhauer résumant les principes de l'idéalisme posés par Kant:

On ne connaît que sa propre intelligence, que soi, seule réalité, le monde spécial et unique que le moi détient, véhicule, déforme, exténue, recrée selon sa personnelle activité; rien ne se meut en dehors du sujet connaissant; tout ce que je pense est réel: la seule réalité, c'est la pensée?.

Entragues le redit encore quand il juge que «le monde matériel et inconscient ne vit et ne se meut que dans l'intelligence qui le perçoit et le recrée à nouveau selon des formes personnelles» (p. 163).

Ce qui se traduit ainsi dans le roman, à travers le retour à Descartes par l'intermédiaire de Schopenhauer, voire, plus généralement, sous l'impulsion de l'idéalisme allemand des systèmes kantien, hégélien et schopenhauerien, c'est donc le doute ontologique fondamental par rapport au réel et le désir d'une vérification systématique de cette lecture du réel conforme aux principes de l'idéalisme; c'est encore et toujours la réévaluation de la pensée individuelle. Elle produit chez le protagoniste de Sixtine une forme exaspérée d'individualisme:

Mais déjà, un peu à ma volonté, le monde s'absentait de moi et par une lente et soudaine recréation, je me refaisais une vie plus harmonieuse à mon sens intime (...). Avec tel parti pris, avec ce système kantien, qui se peut dénommer égoïsme transcendant, ma vie a marché d'un pas relativement léger (p. 62).

Par ses réflexions continuelles ainsi que par ses agissements, Hubert d'Entragues apparaît dans le roman comme principal porteur des idées propres à Schopenhauer. Il se révèle en même temps comme un véritable pessimiste, toujours au sens schopenhauerien du mot. La philosophie de Schopenhauer lui permet de reconnaître l'essentiel de la vie dans l'ennui, la répétition et la souffrance causée par le vouloir-vivre:

J'ai honte de l'avouer, tant cette maladie est banale: je m'ennuie. J'ai des réveils déchirants. Je ne crois rien et je n'aime pas (p. 67).

Il ne cesse de dénoncer l'absence de but dans la vie:

Les trains ont un but; la vie n'en a pas. Mais c'est précisément l'originalité de la vie de n'en pas avoir, de but,

note-t-il dans son carnet d'écrivain (p. 59). En marchant dans les rues de Paris, il médite encore sur l'inutilité de la vie:

Tout est inutile (...). L'inutilité de ma vie n'est pas unique: elle se confond avec l'universel néant (p. 119).

${ }^{9}$ R. de G our mon t, L'Idéalisme, Mercure de France, Paris 1893, p. 13. 
Par conséquent, toute action humaine lui semble inutile:

Pauvres inconscientes abeilles, pour qui travaillez-vous? L'espèce? Mais l'intelligence de quelqu'un balance-t-elle l'universelle sottise? (p. 61).

Son ironie s'attaque jusqu'au sentiment d'espérance:

je ne sais, ne m'étant jamais livré à ses tromperies, si l'espérance n'est autre chose qu'un sanglant éperon, éperonnant l'homme vers un néant futur (p. 62).

De même, il partage les idées de Schopenhauer en matière de douleur:

La douleur est inévitable, mais, loin d'être mauvaise, elle est l'honneur même de l'humanité et la suprême raison de l'existence (p. 182).

Enfin, la définition qu'il donne de l'écrivain, avec son mélange de pessimisme et d'orgueil, porte incontestablement elle aussi la marque du philosophe allemand:

Mon métier est triste: c'est d'expérimenter toutes les douleurs et toutes les horreurs de l'âme humaine, afin que les hommes se reconnaissent dans mon oeuvre et disent: Bien rugi, lion! (...). Tel est mon caractère: on voit que je ne me suis pas appliqué à éluder la connaissance de moi-même; et pourtant nul ne sait mieux que moi à quel point cette science est puérile et malsaine (p. 67-69).

Tout cela, résumant en quelque sorte Schopenhauer, conduit Hubert d'Entragues à la conclusion que

l'existence fausse les âmes, nous ne sommes pas faits pour la vie: une tromperie nous la donne, une duperie nous la conserve (p. 57).

S'impose le rapprochement avec ce passage de Schopenhauer dans Pensées et fragments:

Celui-là même pour qui l'existence est à peu près supportable, à mesure qu'il avance en âge, a une conscience de plus en plus claire qu'elle est en toutes choses un disappointement, nay, a cheat, en d'autres termes qu'elle a le caractère d'une grande mystification, pour ne pas dire d'une duperie... ${ }^{10}$

Voilà qui porte le héros de Sixtine à reconsidérer le problème du bonheur, qu'il cherche à redéfinir constamment sur la base des conclusions qu'impose le pessimisme idéaliste. La conviction de l'impossibilité objective du bonheur pour l'homme le conduit à se rapprocher de l'idée de noluntas, seule forme de bonheur possible selon Schopenhauer et définie par le philosophe comme absense de désir, car,

tant que nous sommes sous la domination des désirs, sous l'empire de la volonté, tant que nous nous abandonnons aux espérances qui nous pressent, aux craintes qui nous persécutent, il n'est pour nous ni repos ni bonheur durable ${ }^{11}$.

${ }^{10}$ A. Schopenhauer, Pensées et fragments, traduits par J. Bourdeau, Félix Alcan, Paris 1900 , p. $55-56$.

${ }^{11}$ Ibidem, p. 155-156. 
Telle est bien la leçon qui se dégage de l'histoire de Gaétan Solange, racontée par l'écrivain Entragues au chapitre XXXI: cédant à la tentation des plaisirs faciles au sein d'un mariage banal, Gaétan Solange n'en sera pas moins conscient de leur fragilité et finira par en avoir honte (La honte d'être heureux est bien le titre du chapitre). Son histoire met en évidence la médiocrité de l'idéal humain du bonheur et les tristes conséquences de la soumission de l'homme à la volonté de vivre. Ce qui s'exprime également ici, par delà l'ironie qui vise l'aspiration bourgeoise au bonheur fondé sur le bien-être et sur le conformisme, c'est la volonté de l'écrivain d'opposer à un bonheur ainsi défini une attitude du renoncement. Face aux tentations illusoires du monde, l'ascétisme du sage peut seul apporter une réponse valable. Comme l'écrit avec justesse Tiziana Goruppi ${ }^{12}$, à la faiblesse de Gaétan Solange s'oppose symboliquement la force de Hubert, fidèle à la définition méprisante du bonheur donnée par Schopenhauer. Selon le philosophe allemand,

nous n'apprécions pas l'ensemble prospère de nos affaires, et nous n'avons de pensées que pour une minutie insignifiante qui nous chagrine. - Le bien-être et le bonheur sont donc tout négatifs, la douleur seule est positive (...). Tout bien, tout bonheur, toute satisfaction sont négatifs, car ils ne font que supprimer un désir et terminer une peine ${ }^{13}$.

Hubert affirme pour sa part:

Le bonheur me laisse froid et ses régulières perspectives m'attristent (p. 263),

tout en dénonçant ses erreurs anciennes:

Hier je jouissais, oui! dans une parfaite inconscience: il m'a fallu sortir du parterre fleuri pour sentir le parfum des fleurs (p. 256).

Cette affirmation ne sera possible en effet qu'au bout d'un long cheminement. C'est surtout à travers sa propre vie que Hubert - en tant que héros du roman - va dénoncer le caractère illusoire du bonheur terrestre. Son aventure personnelle et les dillemmes qui l'accompagnent, ses agissements et, plus encore, son inaction illustrent le mieux les difficultés de vivre et les interrogations philosophiques qui sont celles de l'homme schopenhauerien.

Le sujet qui représente le monde peut-il connaître?, se demandait Schopenhauer dans Le Monde comme volonté et comme représentation. Désireux de soumettre cette philosophie à l'épreuve du réel, l'auteur de Sixtine demande de son côté: le sujet qui prétend représenter le monde peut-il y vivre? Ce sont là, comme le rappelle André Karátson, les «obscures arcanes de l'idéalisme» auxquels se trouve confronté, selon ses propres termes (p. 163), Hubert d'Entragues ${ }^{14}$.

${ }^{12}$ T. G o r u p p i, Remy de Gourmont: L'idea dell'intellettuale e la crisi del romanzo, Pacini, Pisa 1989, p. 121.

\footnotetext{
${ }^{13} \mathrm{~A} . \mathrm{S} \mathrm{c}$ h o p e $\mathrm{n}$ h a u e r, Pensées et fragments, op. cit., p. 52.

${ }^{14}$ A. Karát s o n, op. cit., p. 109-110.
} 
L'intrigue romanesque de Sixtine est en effet tout entière dominée par la difficulté qu'éprouve le héros d'échapper à la pression de la pensée et de passer à l'acte. Au cours des vacances en Normandie, chez la comtesse Aubry, Hubert d'Entragues rencontre Sixtine Magne, femme évanescente et mystérieuse, dont il s'éprend. Ils se revoient à Paris. Sixtine se laisserait volontiers aimer par lui, mais il a beaucoup de mal à se déclarer; il multiplie ses visites, il revit avec exaltation les minutes passées en sa compagnie, il ne cesse de l'imaginer dans ses rêves, mais il ne se décide jamais au geste qui la ferait céder:

Allons, cela sera pour demain ou pour après-demain: Sixtine est en mon pouvoir. C'est agréable, certainement très agréable. Nous passerons de charmantes soirées. Elle est intelligente, je lui lirai mes manuscrits: j'ai besoin, ça et là, de l'opinion d'une femme (...). Quand la reverrai-je? Aujourd'hui? Non. Demain? Non. Mais je lui écrirai? deux fois par jour. Elle me répondra par de petites phrases brèves et impersonnelles, avec des sous-entendus de raillerie. Je me laisserai railler: étant sûr de mon fait, je le puis. Donc, mardi? Nous verrons... (p. 262).

Ces atermoiements du héros occuperont l'essentiel de la narration. Tantôt, fondant ses espoirs sur les droits de la mutuelle intelligence, il s'estime capable de faire progresser une stratégie sûre pour conquérir la femme; il se résout alors à la séduire tout doucement: «Avec un peu d'advertance et de verbales caresses, je puis acquérir près d'elle une agréable situation anténuptiale» (p. 184). Tantôt, il se fait hésitant:

Si le désir, songea-t-il encore, me laisse, même en pensée, la liberté du choix, à quoi bon aimer, ou bien est-ce que j'aime vraiment? Il me faudrait, peut-être, comme à une femme, une possession pour me délivrer de mes doutes (p. 185-186).

Plutôt que de chercher à fléchir la résistance de la femme, aussi faible soit-elle, il semble se plaire davantage à étudier les effets de l'amour sur sa propre intelligence et à cerner ce qui, dans son for intérieur, l'éloigne des recherches émotionnelles:

J'ai peur qu'après sa première floraison mon tempérament ne se féminise et ne s'efface, rongé par la rouille d'une dévorante indécision. Après mes idées, voilà que j'analyse mes sentiments: l'air va devenir irrespirable (p. 186).

L'atmosphère devient irrespirable, en effet. Le désir physique de Hubert entre constamment en conflit avec son besoin d'idéalité. En homme sensible, il se sent attiré par les charmes de la jeune veuve; en disciple de Schopenhauer, il se demande comment on peut tronquer «l'amour momentané au prix de la vie éternelle» (p. 255). Le philosophe lui a bien appris que les sens ne représentent qu'un piège tendu par la volonté de vivre en vue d'annihiler chez l'individu l'aspiration à la liberté, que «la volupté des sens est l'opposé de l'enthousiasme qui nous ouvre le monde idéal» ${ }^{15}$; analogiquement, Hubert déclare que:

${ }^{15}$ A. S c h o p e n h a u e r, Pensées et fragments, op. cit, p. 128. 
l'endosmose d'amour est irréelle et la tromperie du désir, seule, me fait croire à son possible accomplissement. Je sais que c'est un mensonge, je sais la déception qui m'attend: je serai puni par un effroyable désappointement d'avoir cherché l'oubli de moi-mềme en dehors de moi-même, d'avoir trahi l'idéalité... (p. 156).

Identifiant la chasse au bonheur avec la satisfaction des sens, Hubert la fait coincider avec la lâche soumission de l'homme au désir d'exister; il pourrait dire, lui aussi, que «l'amour a toujours pour fondement un instinct dirigé vers la reproduction de l'espèce» ${ }^{16}$. D'ailleurs, la beauté féminine n'a pas à ses yeux d'existence objective:

Je prétends que les femmes ne sont ni belles ni laides, et que tout leur charme s'irradie de leur sexe; le désir esquisse la beauté et l'amour l'achève. Tel laideron, au sens du vulgaire, a pu revêtir une idéale beauté; telle autre femme que tous jugèrent admirable n'a pas franchi les limbes de l'ébauche, n'yant jamais été aimée (p. 71).

Lorsqu'il affirme la vie cérébrale - et que «tout le reste fut rédigé dans les manuels de physiologie» (p. 278), quand il déclare surtout qu' «il n'y a jamais que la couleur des jarretières qui diffère» (p. 262), il semble résumer encore, en termes libertins, le point de vue longuement expliqué par Schopenhauer, selon qui la vérité prend en amour la forme d'une illusion pour agir sur la volonté de l'homme:

C'est en effet une illusion voluptueuse qui abuse l'homme en lui faisant croire qu'il trouvera dans les bras d'une femme dont la beauté le séduit une plus grande jouissance que dans ceux d'une autre, ou en lui inspirant la ferme conviction que tel individu déterminé est le seul dont la possession puisse lui procurer la suprême félicitée ${ }^{17}$.

Les relations d'Entragues avec Sixtine se ressentent fort de cette conception de la femme et de l'amour. Les sarcasmes qu'il adresse à un certain moment à la veuve sont à cet égard extrêmement éloquents: «Vous m'auriez trompé sur la valeur de la vie (...); j'aurais bu, comme une absinthe éternelle, la fluide illusion de vos yeux glauques et je me serais enchaîné à la vie» (p. 52).

C'est donc très cérébralement qu'Hubert d'Entragues rêvera de Sixtine Magne. Puisque le cerveau représente le monde à l'image du rêve, sa passion ne cessera d'aborder la femme comme une réalité imaginaire ${ }^{18}$. Ses «facultés de vision particulières» lui permettent d'appeler l'être aimé près de lui «par des magies», comme il dit; de quoi donc lui servirait la réalité, quand il a «le rêve et la faculté de se protéiser, de posséder successivement toutes les formes de la vie, tous les états d'âme où l'homme se diversifie?» (p. 63). «Le verbe se déroule en une activité intérieure et tout le possible de la vie s'ouvre à moi!», déclare-t-il à Sixtine (p. 47). Celle-ci ne cache point son ironie:

${ }^{16}$ Ibidem, p. 98.

${ }^{17} \mathrm{~A} . \mathrm{S} \mathrm{c}$ h o $\mathrm{p} \mathrm{n} \mathrm{h}$ a u e r, Le monde comme volonté et comme représentation, Supplément au livre IV, chap. XLIV (La métaphysique de l'amour), op. cit, p. 1295-1296.

${ }^{18}$ A. Ka rát s o n, op. cit., p. 109. 
Alors, toutes les femmes sont à votre merci? Si une femme aimée de vous se dérobait à vos prières, l'imagination vous... vous... suffirait (p. 101).

Mais Hubert, refusant de se contenter de la métaphysique du plaisir qu'il dénonce chez les femmes, voit au-delà

de parallèles rêves s'évertuant, au même moment, vers le même but; résultat: la possession mutuelle à distance. Quel triomphe pour l'amour! quelle ressource pour les amants séparés! (p. 101).

Plus d'une fois, cependant, alors qu'il se croit maître de lui et de l'univers sensible, tout ce monde extérieur dédaigné, preque nié, renaît brusquement en l'image de Sixtine:

Alors reprirent les mêmes lamentations: la crainte, l'espoir, le doute: l'amour composé de ces trois termes, surgissait toujours (...): le serpent mordait sa queue (p. 164).

A de tels moments, Entragues sent le plus «le malheur d'être un homme et de n'être que cela» (p. 273). La scène du plaisir solitaire, curieusement évoquée au chapitre XXXII sous forme de poème en prose, en apporte un commentaire presque ironique. De même que «l'heure charnelle» vécue par Hubert au chapitre XV, lorsque, le désir se faisant trop obsédant, il cherche à s'en défaire chez une prostituée. Tout cela complique considérablement les rapports du héros avec son propre corps. Mais l'épisode de la rencontre avec la prostituée Valentine le confirmera dans l'idée que la jouissance corporelle est entachée de péché, qu'elle constitue la principale arme de séduction de la Volonté de vivre. C'est en désespéré qu'il doit chercher à se libérer de l'emprise du désir, s'appliquant le vers de Dante qu'il connaît bien: «Et sans espoir vivre dans le désir» (p. 191). Car «demander à la vie une station dans le bonheur, c'est accorder trop d'importance au mécanisme des sens, c'est se conformer (...) aux normes de la matière, tandis que la volonté doit tendre vers l'affranchissement» (p. 266-267).

C'est bien autour de ce conflit entre l'idéal et la réalité, entre l'attrait des sens et l'aspiration du sage schopenhauerien à l'ascèse, que se développe dans le roman un étrange jeu, où l'indécision et la perpétuelle analyse des sentiments pratiquée par Entragues se heurtent à l'agacement grandissant de Sixtine. Leurs rencontres constituent, selon l'heureuse formule d'André Karátson, «une série de valses-hésitations cocasses ponctuées par des échecs, car tantôt Hubert manque le moment propice, tantôt il risque sa chance au moment où il n'en a aucune» ${ }^{19}$. Tous deux sont certes capables d'une réelle affection, mais l'ironie qui se cache derrière leurs mots et leurs gestes, et l'attitude par trop cérébrale d'Entragues les tiennent étrangers l'un à l'autre. «Elle le raillait et il capitulait; elle fuyait, il la regardait fuir» (p. 117).

L'on ne s'étonne pas, dans ces conditions, de voir les malentendus s'accumuler entre eux et, en fin de compte, Sixtine lui échapper. C'est l'apparition d'un deuxième

${ }^{19}$ Ibidem, p. 112. 
amoureux qui va précipiter l'échec final. Le prétendant s'appelle Sabas Moscowitch, il est Russe; écrivain lui aussi, il vient en France pour réussir au théâtre. Il est barbu, il est séduisant, il a sur Hubert l'avantage de l'exotisme et d'un tempérament plus ardent. Comble d'ironie, pour narguer sa naïveté qu'il méprise, Hubert lui donne des conseils qui, dans son esprit, devraient desservir son rival auprès de Sixtine:

«Osez, mon ami, osez!» Vous ne connaissez peut-être pas les Françaises, mais croyez-en mon expérience, un peu de viol ne leur déplaît pas, je ne dis pas violence, viol: la main de fer gantée de velours peut jouer en amour un rôle décisif; rien n'éclaire mieux une femme sur ses propres sentiments qu'un baiser qui va jusqu'au bout des baisers (p. 177).

A sa douloureuse surprise, les conseils auxquels il n'a pas daigné souscrire lui-même s'avéreront pourtant efficaces: conquise par la spontanéité du Russe, Sixtine se laissera enlever par lui. Délaissé, Hubert se voit condamné à finir ses jours dans la solitude, voué à tout jamais «à la vie cérébrale».

Le message du roman n'est pas pour autant entièrement négatif: une planche de salut sera tendue au protagoniste. Elle portera encore, est-il besoin de le préciser? la marque de Schopenhauer. Dans sa lettre d'adieu adressée à Entragues depuis Nice, Sixtine se réfère explicitement, et à plusieurs reprises, à ses activités d'écrivain; pour compenser leur aventure d'amour avortée, elle lui propose la métaphore de l'oeuvre d'art:

Adieu. Cela vous fera un roman sans conclusion, à la moderne, - car vous l'écrirez, n'est-ce pas? Si non, à quoi bon? Et ainsi l'ombre fugitive s'arrêtera un instant et les passances vaines se réaliseront - oh! bien relativement - au souffle créateur de l'Art (p. 318).

Eh bien, ce défi romanesque sera relevé par le héros. L'ambition du salut par l'écriture plane d'ailleurs constamment sur l'oeuvre de Gourmont, poussant Hubert d'Entragues à une intense activité créatrice. Si la philosophie de Schopenhauer lui fait reconnaître l'essentiel de la vie dans l'ennui et dans la souffrance, elle lui permet aussi, simultanément, l'espoir de s'en affranchir par l'art ${ }^{20}$. Tout comme l'auteur du Monde comme volonté et comme représentation, le protagoniste de Sixtine sait que l'art peut nous apporter une délivrance et une consolation, qu'il transmue la douleur en un spectacle représenté qui efface, par sa beauté, les peines de la vie, et nous dédommage des douleurs réelles; que cette contemplation pure des choses nous arrache, du moins provisoirement, au cauchemar de l'existence. Il mettra donc, lui aussi, l'oeuvre au-dessus de l'action. Cherchant à ressaisir la logique de ses plaintes, Hubert déclare:

J'en ferai de la littérature, je montrerai comment ce peu de bruit intérieur, qui n'est rien, contient tout, comment, avec l'appui bacillaire d'une seule sensation toujours la même et déformée dès son origine, un cerveau isolé du monde peur se créer un monde (p. 186).

${ }^{20}$ Ibidem, p. 110. 
Nous verrons désormais un roman d'Entragues s'insérer dans le roman de Gourmont. Ce roman dans le roman aura pour titre L'Adorant, il sera la transposition, «sur un mode d'extravagance logique», du «drame que Hubert jouait naïvement avec Sixtine» (p. 120). Histoire d'un prisonnier amourex de ìa statue d'une madone, les chapitres successifs de L'Adorant coincident en effet avec les phases importantes de l'histoire d'Hubert d'Entragues. Au début du roman «intérieur», Guido, le prisonnier, apparaît comme le double positif d'Entragues: contrairement à Hubert, qui a désiré le corps de la femme aimée, Guido sait maintenir son amour dans la sphère de l'imagination. Les rapports d'Entragues avec son double imaginaire traduisent donc simplement à ce moment-là, sur le plan symbolique, l'antagonisme entre la vie et l'art. Cependant, au fur et à mesure que se développe cette expérience de l'art, le héros de L'Adorant entre activement dans l'histoire et remplit une fonction initiatrice, devenant un modèle pour le protagoniste de Sixtine: «Ai-je besoin, pour rêver à des amours, d'avoir serré contre ma chair de la chair aimée? (...). Est-ce que Guido a touché sa madone?» (p. 256-257).

Les gestes de Guido orientent désormais son aventure avec Sixtine; ils lui «montrent» que la dimension imaginaire est la seule possible et authentique en amour. Plus les rencontres de Hubert avec Sixtine se font rares, plus les aventures de l'adorant se substituent à son vécu, trahissant la prédominance croissante de la dimension intérieure, idéale, sur la réalité extérieure. Au moment du départ de Sixtine, en comparant son expérience à celle de son «double», Hubert arrive à la conclusion qu' «il fallait l'aimer de loin, comme Guido aime sa madone» (p. 276). Son erreur, c'est d'avoir trahi l'idéal par la tentation sensuelle, alors que «le contact est destructeur du rêve» (p. 276). Mais la passion de Guido a sublimé la sienne: «Je veux vivre une autre vie, je veux redresser idéalement les infirmités inhérentes à mon état charnel, je veux tromper mon âme sur les misères de mon corps...», déclare-t-il (p. 276).

Le travail de l'écriture parvient ainsi à commander au réel du récit extérieur; l'acte de création artistique, authentique expérience transcendante, transforme la banale aventure d'amour du héros de Sixtine en un itinéraire tout intérieur de l'ascèse mystique, qui s'effectue par l'intermédiaire justement de la fonction «désincarnatrice» et, partant, salvatrice, de la contemplation esthétique ${ }^{21}$. Forme d'ascèse, l'art vient définir «la revanche de l'esprit sur le corps» (p. 277). Et le livre se termine par la satisfaction d'un romancier relisant le dernier chapitre de son livre: «Si la vie m'échappe, la transcendance m'appartient; je l'ai payée assez cher, je l'ai payée du prix de toutes les joies terrestres», affirme Hubert (p. 326). «En perdant Sixtine, il s'est retrouvé» (p. 327) par le moyen de l'oeuvre d'art. On peut certes y voir le triomphe de Schopenhauer, triomphe de l'art sur la vie en tant que tentative désespérée d'échapper au vouloir-vivre. On peut noter en même temps, sur le plan

${ }^{21}$ D'après T. G o r u p p i, Remy de Gourmont: L'idea dell'intellettuale e la crisi del romanzo, op. cit., p. 116-118. 
de la technique romanesque, un remarquable dédoublement des structures narratives qui en résulte, l'une des premières manifestations de ce procédé de différentiation de niveaux de création au sein du roman français. La pratique du roman dans le roman apparaît en effet dans Sixtine comme une audace esthétique majeure dont Gourmont fait preuve sans attendre les grands réformateurs du genre au $\mathrm{XX}^{\mathrm{e}}$ siècle. Mais le problème, à peine signalé par Lucien Dällanbach dans son travail sur le récit spéculaire ${ }^{22}$, mérite d'être étudié de plus près.

\section{BIBLIOGRAPHIE}

Col i n, R.P. (1979), Schopenhauer en France: un mythe naturaliste, Presses Universitaires de Lyon.

Gor up i, T. (1989), Remy de Gourmont: L'idea dell'intellettuale e la crisi del romanzo, Pacini, Pisa.

G o u r m on t, de R. (1982), Sixtine. Roman de la vie cérébrale, suivi de Lettres à Sixtine, Préface d'Hubert Juin, Union Générale d'Éditions, 1982, coll. 10/18, série «Fins de siêcles», Paris.

K a ráts on, A. (1989), Les arcanes de l'idéalisme (Réception esthétique de Schopenhauer dans «Sixtine» de Remy de Gourmont), dans: A. Henry (dir.), Schopenhauer et la création littéraire en Europe, Paris, Klincksieck, Paris.

${ }^{22}$ L. D ä 11 e $\mathrm{n}$ b a c h, Le récit spéculaire. Essai sur la mise en abyme, Seuil, Paris 1977, p. 29. 